\title{
Nonionic Surfactant Solubilization of PAHs in Bentonite/Aqueous Systems
}

\author{
Masakazu NIINAE ${ }^{1}$, Hiroaki KIYOTO² and Kenji AOKI ${ }^{1}$
}

\author{
${ }^{1}$ Department of Urban and Environmental Engineering, Kyoto University, Kyoto 615-8540, Japan \\ ${ }^{2}$ Graduate School of Engineering (Present at Japan Patent Office, Tokyo 100-8915, Japan)
}

\begin{abstract}
Enhanced solubilization of phenanthrene and pyrene from bentonite in micellar solution of Brij 30 and Triton X-100 has been studied. Experiments in batch bentonite/aqueous system were conducted to investigate the effectiveness of solubilization of phenanthrene and pyrene. The results obtained in this study are as follows:

The solubilization of pyrene from bentonite in surfactant solutions was very low because of low water solubility and strong sorption onto bentonite of pyrene. Meanwhile, the solubilization of phenanthrene increased at bulk surfactant concentrations greater than the surfactant critical micelle concentration and was in order of Triton X-100>Brij 30. The solubilization of phenanthrene was also analyzed based on the partitioning of phenanthrene between bentonite, nonionic surfactant micelle and aqueous solution surrounding the micelles.
\end{abstract}

Key words: Nonionic surfactant, PAHs, Enhanced solubilization, Bentonite

\section{Introduction}

Polycyclic aromatic hydrocarbons (PAHs) are formed by natural and anthropogenic pyrolysis of organic matter during forest fires, fossil fuel utilization, and chemical manufacture. Most PAHs are carcinogenic, which causes environmental concerns. PAH removal from soils and aquifers by natural attenuation mechanisms or traditional remediation efforts such as pump-and-treat is slow due to the low solubility of these compounds in water. It is well-known that surfactants can increase the solubility of a hydrophobic organic compound (HOC, low water solubility) by partitioning it into the hydrophobic cores of surfactant micelles ${ }^{1}$. Generally, surfactants can be divided into three categories based on their hydrophilic head groups: nonionic, anionic and cationic. In soil remediation applications, loss of surfactant is a major concern as it not only reduces the effectiveness of the surfactant but also increases the operational cost by higher dose requirements. Sorption of surfactants depends on the nature of the soil surface and the $\mathrm{pH}$ in the system. Anionic surfactants usually have a low susceptibility for sorption because most soil surfaces are negatively

Accepted 8 December 2006 charged. However, higher adsorption of anionic surfactant to soil usually is observed at lower $\mathrm{pH}$ because the soil/liquid interface is more positively charged at low $\mathrm{pH}$. In contrast, cationic surfactant losses often are significant, making their use for remediation less favorable compared with anionic and nonionic surfactants ${ }^{2}$. Nonionic surfactants may have specific advantages compared to anionic or cationic surfactants in regard to certain aspects of engineered remediation of contaminated soils because of differences in surfactant charge, critical micelle concentration (CMC) range, toxicity, and biodegradability ${ }^{3)}$. The extent of sorption of HOC is known to be dependent on soil characteristics such as clay mineralogical composition and concentrations ${ }^{3)}$. However, comparatively little has been published about the surfactant solubilization of HOC from clay/aqueous systems $\mathrm{s}^{4,5}$.

The objective of this research is to investigate the nonionic surfactant solubilization of PAHs from clay/aqueous system.

\section{Materials and Methods}

PAHs used in this study were phenanthrene $\left(\mathrm{C}_{14} \mathrm{H}_{10}\right)$ and pyrene $\left(\mathrm{C}_{16} \mathrm{H}_{10}\right)$. These PAHs were obtained from Nacalai Tesque and were all analytical reagent grade. Nonionic surfactants used in 
this study were Brij $30\left(\mathrm{C}_{12} \mathrm{H}_{25}\left(\mathrm{CH}_{2} \mathrm{CH}_{2} \mathrm{O}\right)_{4} \mathrm{OH}\right.$, dodecylethoxylate with 4 ethoxylate units, average molecular weight: 363, Aldrich Chemical Company) and Triton X-100 $\left(\mathrm{C}_{8} \mathrm{H}_{17}-\mathrm{C}_{6} \mathrm{H}_{4}\right.$ $\mathrm{O}\left(\mathrm{CH}_{2} \mathrm{CH}_{2} \mathrm{O}\right)_{x} \mathrm{H}$, octylphenylethoxylate with average $x=9.5$, average molecular weight: 628 , Aldrich Chemical Company), and were used without further purification. Also, Na-bentonite (Oe, Yamagata, Japan) was used as a clay mineral.

Surface tension experiments to evaluate the critical micelle concentration of surfactant in soil/ aqueous system and aqueous system were conducted with tensiometer (Kyowa Interface Science CBVP-A3). Batch test samples were consisted of $1.0 \mathrm{~g}$ of bentonite and $50 \mathrm{~cm}^{3}$ of surfactant solutions of varying concentrations in soil/aqueous systems, and $50 \mathrm{~cm}^{3}$ of surfactant solutions of varying concentrations in aqueous systems, all of which were in a $50 \mathrm{~cm}^{3}$ glass centrifuge tube. After the centrifuge tubes were sealed, they were shaking in the water bath maintained at $298 \mathrm{~K}$ for $5 \mathrm{hr}$ before measurements of surface tension were made. In the soil/aqueous systems, the measurements of surface tension were made for the supernatant from the centrifuged soil/aqueous systems.

In batch tests for solubilization of PAH in the soil/aqueous systems, $1.0 \mathrm{~g}$ of bentonite and $50 \mathrm{~cm}^{3}$ of methanol solution dissolved phenanthrene or pyrene were added to $50 \mathrm{~cm}^{3}$ centrifuge tube and they were shaking in the water bath maintained at $298 \mathrm{~K}$ for $3 \mathrm{hr}$. After the centrifugation, bentonite and bulk solutions were separated. $50 \mathrm{~cm}^{3}$ of surfactant solutions of varied concentrations was added to each tube, after methanol was allowed to evaporate from bentonite. Tubes were shaking in the water bath maintained at $298 \mathrm{~K}$ for $48 \mathrm{hr}$ and they were then centrifuged to separate bentonite from bulk solutions.

A Shimadzu high-pressure liquid chromatograph (detector: UV, column: VP-ODS, mobile phase: acetonitrile $(70 \mathrm{vol} \%)$ : water $(30 \mathrm{vol} \%))$ was used to quantify phenanthrene and pyrene concentrations in the sample solutions from the batch tests.

Solubilization $(S)$ of PAH achieved by surfactant washing can be determined using certain experimentally measured values, as shown below:

$$
\text { Solubilization }(S)=C_{\text {aq,tot }} / q_{\text {soil }}^{0} W
$$

where $C_{\text {aq,tot }}\left(\mathrm{mol} / \mathrm{dm}^{3}\right)$ is the total concentration of $\mathrm{PAH}$ in the surfactant solution, $q_{\text {soil }}^{0}(\mathrm{~mol} / \mathrm{g})$ is the initial concentration of PAH in bentonite and $W$ is the mass of bentonite per volume of solution $(\mathrm{g} /$ $\left.\mathrm{dm}^{3}\right)$.

\section{Results and Discussion}

\subsection{Sorption of nonionic surfactant onto ben- tonite}

Figs. 1 and 2 show the surface tension curves associated with Brij 30 and Triton X-100. The curve on left is for an aqueous solution of nonionic surfactant and the curve on the right is for the supernatant of bentonite/aqueous system. Surface tension is plotted as a function of the logarithm of the surfactant concentration $\left(\mathrm{mol} / \mathrm{dm}^{3}\right)$, which represents the total number of moles of surfactant added to the system per litter of solution. The point of inflection in each surface tension curve denotes a value for the logarithm of $D_{\mathrm{cmc}}(\mathrm{mol} /$ $\mathrm{dm}^{3}$ ), the surfactant concentration at which the aqueous-phase CMC $\left(\mathrm{mol} / \mathrm{dm}^{3}\right)$ is attained. In the aqueous system, there is no sorption of surfactant, and $D_{\text {cmc,aq }}\left(\mathrm{mol} / \mathrm{dm}^{3}\right)$ is equivalent to the aqueousphase CMC. In the bentonite/aqueous system, however, a significant fraction of the surfactant may sorb onto bentonite. The concentration at which the aqueous CMC is attained in such a system, $D_{\text {cmcsoil }}$, is the sum of the aqueous-phase CMC $\left(D_{\text {cmc,aq }}\right)$ and $C_{\text {sorb,cmc }}\left(\mathrm{mol} / \mathrm{dm}^{3}\right)$, the sorbed surfactant concentration in moles per litter, evaluated at the aqueous phase CMC:

$$
D_{\text {cmc,soil }}=D_{\text {cmc,aq }}+C_{\text {sorb,cmc }}
$$

As a result of sorption of surfactant onto bentonite, the value of $D_{\text {cmcsoil }}$ is seen from Figs. 1 and 2 to be about an order of magnitude greater than $D_{\text {cmc,aq }}$. The two values of $D_{\text {cmcsoil }}$ and $D_{\text {cmc,aq }}$ for

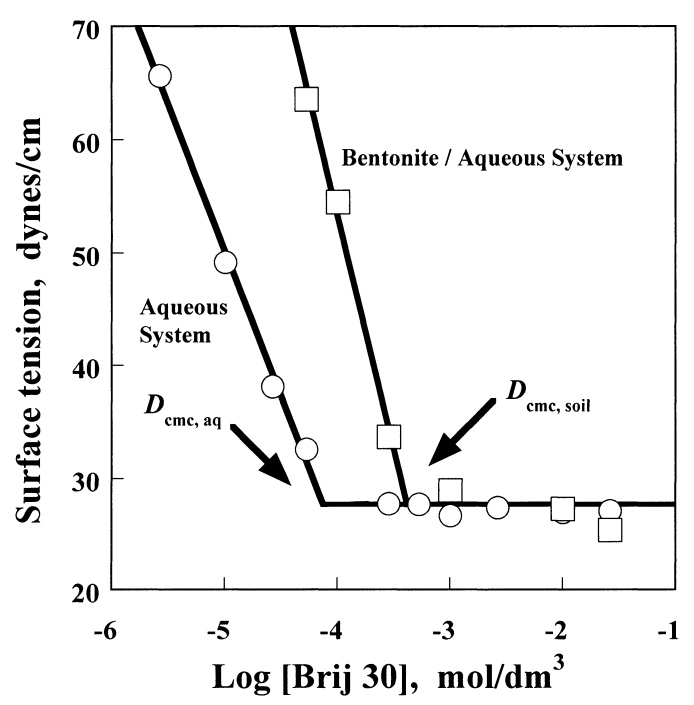

Fig. 1 Relationship between surface tension and Brij 30 concentration in aqueous and bentonite/aqueous systems. 


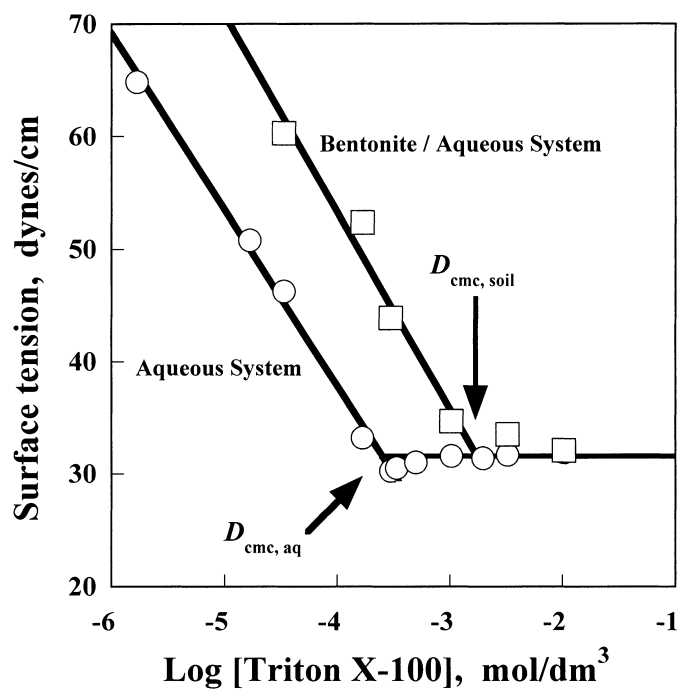

Fig. 2 Relationship between surface tension and Triton $\mathrm{X}-100$ concentration in aqueous and bentonite/ aqueous systems.

Brij 30 are $4.15 \times 10^{-4} \mathrm{~mol} / \mathrm{dm}^{3}$ and $7.47 \times 10^{-5}$ $\mathrm{mol} / \mathrm{dm}^{3}$, respectively. Meanwhile, the two values of $D_{\text {cmcsoil }}$ and $D_{\text {cmc,aq }}$ for Triton X-100 are $1.73 \times$ $10^{-3} \mathrm{~mol} / \mathrm{dm}^{3}$ and $2.51 \times 10^{-4} \mathrm{~mol} / \mathrm{dm}^{3}$, respectively.

\subsection{Solubilization of phenanthrene and pyrene}

Fig. 3 shows the experimental data for the nonionic surfactant solubilization of phenanthrene in the bentonite/aqueous system. The solubilization of phenanthrene is low at concentrations below $D_{\text {cmc,soil }}$ and significantly increases at the concentrations greater than $D_{\text {cmcsoil. }}$ The increase in solubilization of phenanthrene at the concentrations greater than $D_{\text {cmc,soil }}$ is due to the form of micelles. The nonionic surfactant micelles solubilize phenanthrene in their hydrophobic interiors, and in so doing, increase the solubilization of phenanthrene in the bulk solution. Fig. 4 shows the experimental data for the nonionic surfactant solubilization of pyrene in the bentonite/aqueous system. The solubilization of pyrene is very low at the concentrations below $D_{\text {cmcsoil }}$ and slightly increases at the concentrations greater than $D_{\text {cmcsoil }}$.

As shown in Figs. 3 and 4, the nonionic surfactant solubilization of pyrene in the bentonite/aqueous system is lower than that of phenanthrene. The equilibrium PAH partitioning between the sorbed phase and the aqueous phase is characterized by $K_{\mathrm{d}, \mathrm{cmc}}\left(\mathrm{dm}^{3} / \mathrm{g}\right)$, the ratio of the number of moles of PAH sorbed per gram of clay mineral to the number of moles of PAH dissolved per litter of surfactant solution at $D_{\mathrm{cmc} \text {,soil }}$.

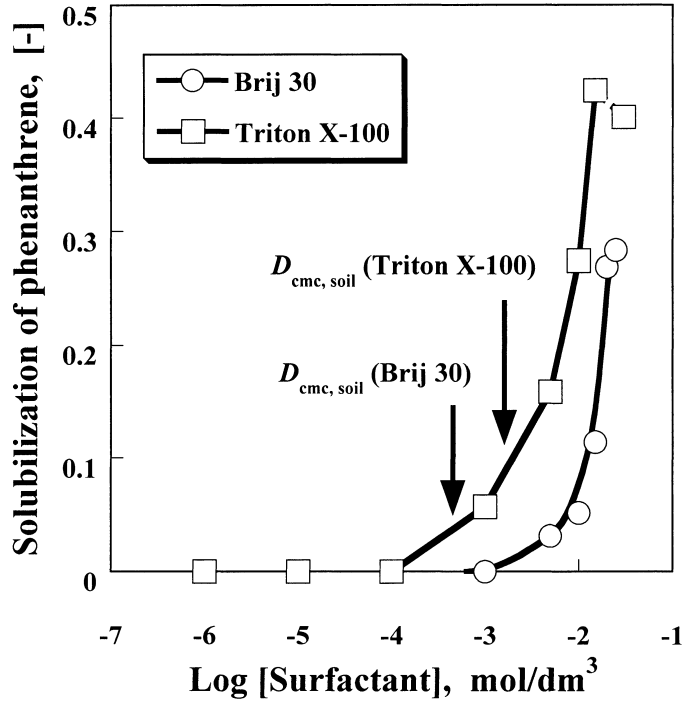

Fig. 3 Relationship between solubilization of phenanthrene and nonionic surfactant concentration.

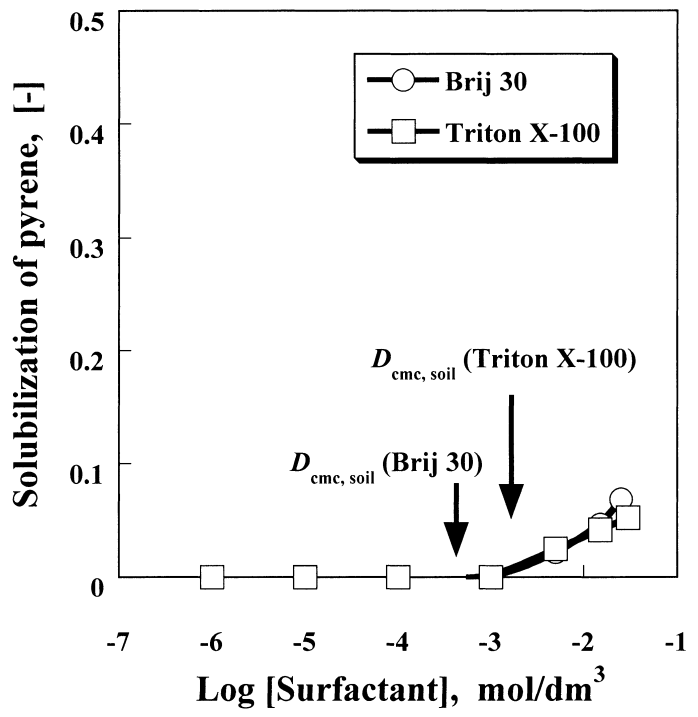

Fig. 4 Relationship between solubilization of pyrene and nonionic surfactant concentration.

$$
K_{\mathrm{d}, \mathrm{cmc}}=\left(n_{\mathrm{sorb}, \mathrm{cmc}} / w_{\text {soil }}\right) /\left(n_{\mathrm{aq}, \mathrm{cmc}} / v_{\mathrm{aq}}\right)
$$

where $n_{\text {sorb,cmc }}(\mathrm{mol})$ is the equilibrium number of moles of PAH sorbed onto clay at $D_{\text {cmc,soil }}, w_{\text {soil }}(\mathrm{g})$ is the weight of clay, $v_{\mathrm{aq}}\left(\mathrm{dm}^{3}\right)$ is the volume of the aqueous solution, and $n_{\mathrm{aq}, \mathrm{cmc}}(\mathrm{mol})$ is the equilibrium number of moles of PAH in surfactant solution at $D_{\text {cmcssoil. }}$ Also, the equilibrium PAH distribution between the micellar phase and aqueous phase can be characterized by a partition coefficient, $K_{\mathrm{m}}(-)$, which represents the ratio of $X_{\mathrm{m}}$, the mole fraction 
of PAH in the micellar phase, to $X_{\mathrm{a}}$, the mole fraction of PAH in the aqueous phase.

$$
K_{\mathrm{m}}=X_{\mathrm{m}} / X_{\mathrm{a}}
$$

For example, the values of $K_{\mathrm{m}}$ of pyrene and phenanthrene with Brij 30 are $3.39 \times 10^{6}$ and $3.72 \times 10^{5}$, respectively, and the value of $K_{\mathrm{m}}$ of pyrene is greater than that of phenanthrene ${ }^{3)}$. These data indicate that the distribution of pyrene from the aqueous phase to the micellar phase is greater than that of phenanthrene. However, the nonionic surfactant solubilization of pyrene in the bentonite/aqueous system is lower than that of phenanthrene. It is assumed that the value of $K_{\mathrm{d}, \mathrm{cmc}}$ of pyrene is significantly greater than that of phenanthrene, therefore, sorption of pyrene onto bentonite is stronger than that of phenanthrene and the effect of $K_{\mathrm{d}, \mathrm{cmc}}$ is stronger compared with $K_{\mathrm{m}}$. Pyrene having four benzene rings is a more hydrophobic compound compared to phenanthrene having three benzene rings, therefore, the solubility of pyrene in water is lower than that of phenanthrene (solubility of phenanthrene: $7.2 \times 10^{-6} \mathrm{~mol} / \mathrm{dm}^{3}$, solubility of pyrene: $\left.6.8 \times 10^{-7} \mathrm{~mol} / \mathrm{dm}^{3}\right)^{3)}$ and the sorption of pyrene to bentonite organic matter is stronger than that of phenanthrene. Also, clay minerals containing montmorillonite of main component of bentonite have a high negative surface charge and produce an electric field in the solution by double layer formation, and the electrical attraction of PAH to mineral sites as a result of the electric field is considerably stronger than the surface forces acting between organic matter and PAH. Therefore, the presence of four benzene rings in pyrene results in high charge density of the molecule, it is likely strongly bonded by electrostatic attraction ${ }^{5}$. The nonionic surfactant solubilization of pyrene in the bentonite/aqueous system was very low, therefore, the subsequent investigation was just performed for the nonionic surfactant solubilization of phenanthrene in the bentonite/aqueous system.

By considering PAH partitioning in the bentonite/aqueous system, solubilization $(S)$ can be expressed as:

$$
S=\left(n_{\text {aq, cmc }}+n_{\text {mic,surf }}\right) /\left(n_{\text {aq,cmc }}+n_{\text {mic,surf }}+n_{\text {sorb,surf }}\right)(5)
$$

where $n_{\text {mic,surf }}(\mathrm{mol})$ is the number of moles of PAH in micelle and $n_{\text {sorb,surf }}$ is the number of moles of PAH sorbed onto bentonite in the presence of surfactant. Also, $n_{\text {mic,surf }}$ and $n_{\text {sorb,surf }}$ can be expressed as follows:

$$
\begin{aligned}
n_{\text {mic,surf }}= & K_{\mathrm{m}} V_{\mathrm{w}} C_{\mathrm{aq}}\left(m_{\mathrm{tot}}-Q_{\mathrm{max}} w_{\text {soil }}-v_{\mathrm{aq}} D_{\mathrm{cmc}, \mathrm{aq}}\right) \\
& /\left(1-K_{\mathrm{m}} V_{\mathrm{w}} C_{\mathrm{aq}}\right)
\end{aligned}
$$

$$
n_{\text {sorb,surf }}=K_{\mathrm{d}, \mathrm{cmc}} C_{\mathrm{aq}} w_{\text {soil }}
$$

where $V_{\mathrm{w}}$ is molar volume of water $\left(1.805 \times 10^{-2}\right.$ $\left.\mathrm{dm}^{3} / \mathrm{mol}\right), C_{\text {aq }}\left(\mathrm{mol} / \mathrm{dm}^{3}\right)$ is PAH concentration in aqueous solution, $m_{\text {tot }}$ is the total number of moles of surfactant supplied to the system and $Q_{\max }(\mathrm{mol} /$ $\mathrm{g}$ ) is the maximum number of moles of nonionic surfactant sorbed per unit weight of bentonite. $Q_{\max }$ can be expressed by the following equation and can be obtained from the relationship between nonionic surfactant concentration and surface tension (Brij 30: $1.70 \times 10^{-5} \mathrm{~mol} / \mathrm{g}$, Triton X-100: $\left.7.40 \times 10^{-5} \mathrm{~mol} / \mathrm{g}\right)$.

$$
Q_{\text {max }}=C_{\text {sorb,cmc }} v_{\text {aq }} / w_{\text {soil }}=\left(D_{\text {cmc,soil }}-D_{\text {cmc,aq }}\right) v_{\text {aq }} / w_{\text {soil }}
$$

By incorporation of equations (6) and (7) to equation (5), the following equation can be derived.

$$
\begin{aligned}
S= & \left(1+K_{\mathrm{m}} V_{\mathrm{w}} m_{\mathrm{mic}} / v_{\mathrm{aq}}\left(1-K_{\mathrm{m}} V_{\mathrm{w}} C_{\mathrm{aq}}\right)\right) \\
& /\left(1+K_{\mathrm{m}} V_{\mathrm{w}} m_{\mathrm{mic}} / v_{\mathrm{aq}}\left(1-K_{\mathrm{m}} V_{\mathrm{w}} C_{\mathrm{aq}}\right)\right. \\
& \left.+K_{\mathrm{d}, \mathrm{cmc}} w_{\text {soil }} / v_{\mathrm{aq}}\right)
\end{aligned}
$$

where $m_{\text {mic }}(\mathrm{mol})$ is expressed as:

$$
m_{\text {mic }}=m_{\text {tot }}-Q_{\text {max }} w_{\text {soil }}-v_{\text {aq }} D_{\text {cmc,aq }}
$$

If $K_{\mathrm{m}} V_{\mathrm{w}} C_{\mathrm{aq}}$ is negligible compared with unity, as appears to be the case for PAH having the values of octanol/water partition coefficient $\left(K_{\text {ow }}(-)\right)$ greater than about $1.0 \times 10^{4}\left(K_{\text {ow }}\right.$ value of phenanthrene is $\left.3.7 \times 10^{4}\right)^{6}$, then equation (9) can be approximated as follows:

$$
\begin{aligned}
S= & \left(1+K_{\mathrm{m}} V_{\mathrm{w}} m_{\mathrm{mic}} / v_{\mathrm{aq}}\right) / \\
& \left(1+\left(K_{\mathrm{m}} V_{\mathrm{w}} m_{\mathrm{mic}}+K_{\mathrm{d}, \mathrm{cmc}} w_{\text {soil }}\right) / v_{\mathrm{aq}}\right)
\end{aligned}
$$

The following equation can be derived from equation (11).

$$
\begin{aligned}
S /(1-S)= & \left(1+K_{\mathrm{m}} V_{\mathrm{w}}\left(D_{\mathrm{s}}-C_{\mathrm{sorb}, \mathrm{cmc}}-D_{\mathrm{cmc}, \mathrm{aq}}\right)\right) \\
& / K_{\mathrm{d}, \mathrm{cmc}} w_{\mathrm{soil}} / v_{\mathrm{aq}}
\end{aligned}
$$

where $D_{\mathrm{s}}\left(\mathrm{mol} / \mathrm{dm}^{3}\right)$ is the total concentration of surfactant added to the system. It is evident from equation (12), a plot of experimental values for $[S /$ $(1-S)]$ against experimental values for the expression $\left(D_{\mathrm{s}}-C_{\text {sorb,cmc }}-D_{\text {cmc,aq }}\right)$ should be linear. Since the slope of the line is equal to $\left\{\left(K_{\mathrm{m}} V_{\mathrm{m}}\right) /\right.$ $\left.\left[K_{\mathrm{d}, \mathrm{cmc}}\left(w_{\text {soil }} / v_{\mathrm{aq}}\right)\right]\right\}$, the slope of the plotted data provides a reasonable estimate for the value of the ratio $K_{\mathrm{m}} / K_{\mathrm{d}, \mathrm{cmc}}$. Fig. 5 shows the values of $[S /(1-S)]$ plotted against the values of $\left(D_{\mathrm{s}}-C_{\text {sorb,cmc }}-D_{\text {cmc,aq }}\right)$ for solubilization of phenanthrene with Triton X100. Similar result was obtained for the solubilization of phenanthrene with Brij 30. $K_{\mathrm{d} \text {,cmc }}$ value of $19.7\left[\mathrm{dm}^{3} / \mathrm{g}\right]$ for Brij 30/phenanthrene and $K_{\mathrm{d}, \mathrm{cmc}}$ of $12.8\left[\mathrm{dm}^{3} / \mathrm{g}\right]$ for Triton X-100/phenanthrene can 


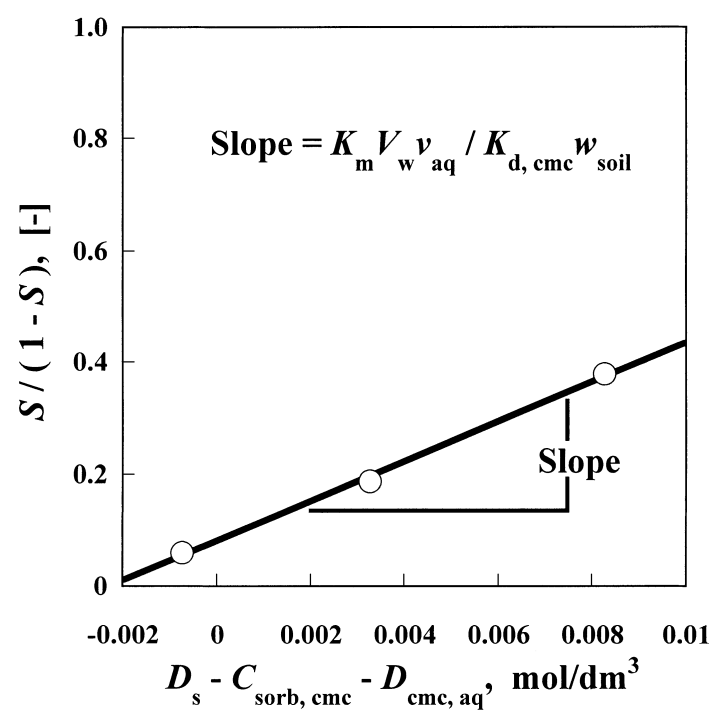

Fig. 5 Plot of $S /(1-S)$ versus $\left(D_{\mathrm{s}}-C_{\text {sorb,cmc }}-D_{\text {cmc,aq }}\right)$.

be estimated, respectively, using $K_{\mathrm{m}}$ value of $3.72 \times 10^{5}$ for Brij 30/phenanthrene ${ }^{3)}$ and $K_{\mathrm{m}}$ value of $5.01 \times 10^{5}$ for Triton X-100/phenanthrene ${ }^{3)}$. Therefore, solubilization $(S)$ may be estimated using equation (11). Figs. 6 and 7 show the plots of the experimental $S$ values and the estimated $S$ values based on equation (11) for Brij 30/phenanthrene and Triton X-100/phenanthrene, respectively. It is apparent that Figs. 6 and 7 show the relatively good agreement between the experimental data and the solubilization values calculated for these micelle-forming nonionic surfactant systems using equation (11). The solubilization of phenanthrene is in order of Triton X-100>Brij 30 .

\section{Conclusions}

Enhanced solubilization of phenanthrene and pyrene from bentonite in micellar solution of Brij 30 and Triton X-100 has been studied and the following results are drawn:

(1) The solubilization of pyrene from bentonite in surfactant solutions is very low because of low water solubility and strong sorption onto bentonite of pyrene.

(2) The solubilization of phenanthrene increases at bulk surfactant concentrations greater than $D_{\text {cmc,soil. }}$

(3) The solubilization of phenanthrene is analyzed based on the partitioning of phenanthrene between bentonite, nonionic surfactant micelle and aqueous solution surrounding the micelles, and the experimental data are relatively consistent with the estimated solubilization

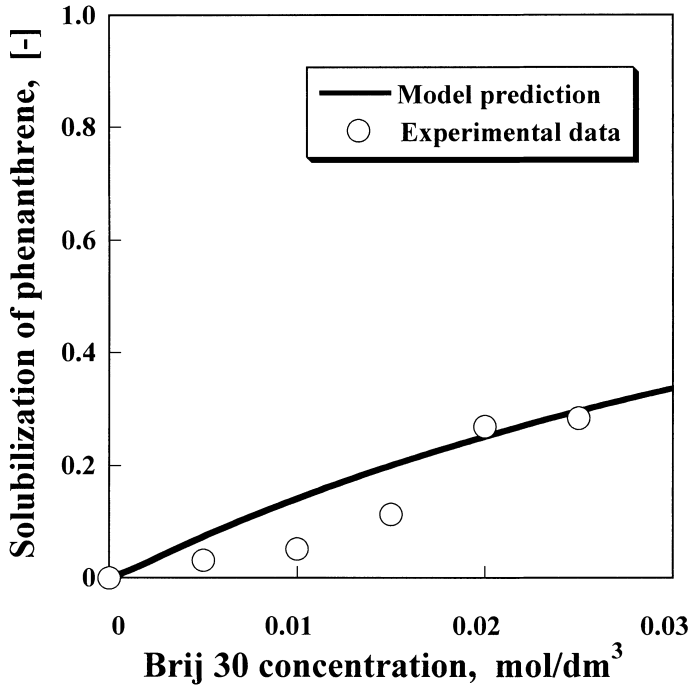

Fig. 6 Relationship between solubilization of phenanthrene and Brij 30 concentration.

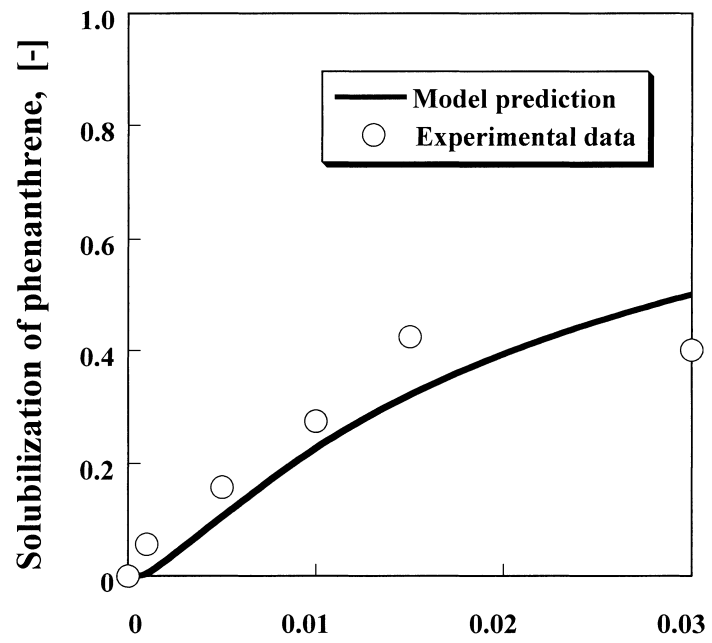

Triton X-100 concentration, $\mathrm{mol} / \mathrm{dm}^{3}$

Fig. 7 Relationship between solubilization of phenanthrene and Triton X-100 concentration.

values.

(4) The solubilization of phenanthrene is in order of Triton X-100>Brij 30.

\section{References}

1) S. Guha, P.R. Jaffe, C.A. Peters: Environmental Science and Technology, 32, pp.930935 (1998)

2) C. Huang, J.E.V. Benschoten, T.C. Healy, M.E. Ryan: Journal of Soil Contamination, 
6, pp. 537-556 (1997)

3) D.A. Edwards, R.G. Luthy, Z. Liu: Environmental Science and Technology, 25, pp. 127133 (1991)

4) E. Klumpp, H. Heitmann, H. Lewandowski, M.J. Schwuger: Progress in Colloid and
Polymer Science, 89, pp. 181-185 (1992)

5) D.R. Ghosh, T.M. Keinath: Environmental Progress, 13, pp. 51-59 (1994)

6) S.W. Karickhoff, D.S. Brown, T.A. Scott: Water Research, 13, pp. 241-248 (1979) 